\title{
Is childhood dental anxiety a result of irregular attendance?
}

The relationship between anxiety and dental treatment experience in 5-year-old children.

K. M. Milsom, M. Tickle, G. M. Humphris and A. S. Blinkhorn Br Dent J 2003; 194: 503-506

\section{Objectives}

To examine the relationship between dental anxiety, dental attendance and past treatment history in 5-year-old children after taking into account confounding influences.

\section{Methods}

A cross sectional study of all 5-year-old children living in Ellesmere Port and Chester. All children were clinically examined and $\mathrm{dmft}$ and its components were recorded. A postal questionnaire was sent to parents of participating children to identify whether children attended the dentist on a regular asymptomatic basis or only when experiencing problems. Additionally parents were asked to judge whether they and their child were anxious about dental treatment. The socio-economic status of the family was measured using the Townsend Material Deprivation Index of the electoral ward in which they resided. The bivariate relationships between anxiety and reported attendance, caries experience, past extraction and restoration history were assessed using chisquare and $t$-tests. Multiple logistic regression analyses were used to identify predictors for dental anxiety.

\section{Results}

A total of 1,745 children received both a clinical examination and a questionnaire and 1,437 parents responded, a response rate of $82.3 \%$. One in ten parents (10.8\%) judged their child to be dentally anxious. Anxious children had significantly $(\mathrm{p}<0.001)$ more caries experience (dmft 2.58 vs 1.12). Multiple logistic regression analyses confirmed that anxious children were more likely to be irregular attenders (OR 3.33, 95\% CI 2.22, 5.00), have anxious parents (OR 1.60, 95\% CI 1.09, 2.36), and to have undergone dental extraction in the past (OR 3.50, 95\% CI 2.10, 5.85), after controlling for gender and socio-economic status. A past history of restoration was not a significant predictor of anxiety after controlling for other factors.

\section{Conclusions}

Dental anxiety is a fairly common condition in 5-year-old children in the North West of England. It is closely associated with a symptomatic, irregular attendance pattern, a history of extraction and having a dentally anxious parent. The cause and effect dynamics of these relationships need to be determined.

\section{COMMENT}

The conduct of research is both an academic and a practical exercise which demands the development of conceptual and theoretical frameworks to understand the findings of research as well as the identification of how these frameworks provide solutions to the problems faced by practitioners. The paper by Milsom and colleagues is an elegant demonstration of this balance. Dental anxiety in children is a common and potentially distressing problem for the child, their parents and the treating dental practitioner. A simple model of the development of dental anxiety suggests that the experience of traumatic events or treatments in the surgery should be associated with the development of fear through the learning of an association of pain and distress with the dental setting. This is termed 'direct conditioning.' An extension to this theory suggests that if a child has a number of positive experiences at the dentist they will learn a positive attitude toward the dentist. If these positive experiences are then followed by some traumatic event then the ability of the trauma to produce dental fear will be inhibited by the prior learning ('latent inhibition'). Previous research has sought to identify the extent to which these putative mechanisms can be observed to operate in the population, and it is to this debate that Milsom etal. contribute so significantly. The survey they describe is remarkable in that it covers an entire population of 5-year-olds in a defined geographic area, and achieves a very high response rate. Evidence is found to support both the direct conditioning of fear and latent inhibition. The practical implications are clear - regular attendance and positive experiences in the dental setting are to be encouraged and will be associated with decreased dental fear. As with all good research this leads to more questions; how can regular attendance be encouraged amongst parents who may themselves be fearful of the dentist, and how can systems of remuneration be identified to support a preventive approach which incorporates regular attendance for children and activities that may be time consuming for the general dental practitioner?

\section{J. T. Newton, \\ Professor of Psychology as applied to Dentistry}

\section{IN BRIEF}

- Dentally related anxiety occurs in about $10 \%$ of the population of 5 year-old children and is related to irregular attendance, traumatic treatment procedures and the anxiety of the child's parents.

- Children who are sporadic attenders, usually present in pain and therefore often require an extraction and extraction is associated with anxiety. This pattern of attendance and care can set up a vicious cycle leading to long lasting dental anxiety problems.

- To prevent anxiety, we must break this cycle by making the dental experience easier for parents and more pleasant for children, but also by providing effective public health measures to prevent dental caries.

- In measuring the outcomes of dental treatment provided to children, we must measure not only clinical dental factors such as the longevity of restorations, perhaps more important is the need to measure the impact of the treatment on the patient's quality of life. 